\title{
Metastatic brain tumor secondary to pancreatic adenocarcinoma
}

Julia Pastorello 1, Emanuela Lando 2*, Marina Ractz Bueno 1, Camila dos Santos do Amaral 1

${ }^{1}$ Department of Clinical Oncology, Hospital de Clínicas de Passo Fundo - HCPF, Passo Fundo, Rio Grande do Sul/ RS, Brazil.

${ }^{2}$ Medical Student, Faculdade Meridional - IMED, Passo Fundo, Rio Grande do Sul/RS, Brazil.

${ }^{*}$ Corresponding author: Emanuela Lando. Paisandú Street, 358. Cep: 99010100 -Passo Fundo, Rio Grande doSul/RS. Brasil. Phone: +55 (54) 9 91861467. E-mail: manu.lando@hotmail.com.

Research Ethics Committee Approval (if necessary): We declare that the patient or legal representant approved the study by signing an informed consent form and the study followed the ethical guidelines established by the Declaration of Helsinki.

Received on: Dec 26, 2021. Accepted on: Jan 1, 2022. Available online: Jan 17, 2022.

\section{Abstract}

Pancreatic neoplasm is represented by the subtype Adenocarcinoma in $90 \%$ to $95 \%$ of cases, characterized by the high lethality rate of its presentations; however, cases of progression in the brain site are manifested in only $0.1 \%$ to $0.3 \%$ of all the cases. The present study aims to report a rare case of a patient undergoing treatment for pancreatic cancer, which had a median overall survival significantly higher than the median time related to such neoplasm, and during the final evolution of the disease progression, an unusual associated with symptomatic brain metastatic activity, was evidenced, thus we correlated the current medical literary data to the events presented by the reported patient. Thus, the reported case becomes of extreme clinical relevance for the study of modern clinical oncology.

Keywords: Pancreatic cancer; Gastroenterology; Neuro-oncology; Oncology.

\section{Introduction}

Pancreatic cancer, a neoplastic entity represented by the subtype Adenocarcinoma in $90 \%$ to $95 \%$ of cases, composed of a higher diagnostic prevalence in males over the age of 60 and black ethnicity, a tumor subtype intrinsically associated with somatic causes, especially smoking, obesity and alcoholism and genetic causes [1-7].

According to the National Cancer Institute (NCI), in Brazil such a carcinogenic subtype is responsible for about $2 \%$ of all diagnoses among solid tumors and responsible for $4 \%$ of neoplastic mortality [2]. 
Solid tumor with a high lethality rate composed of similar incidence and mortality, with life expectancy being approximately $5 \%$ in 5 years, the diagnosis in most cases is late, in advanced stages, strengthening the associated metastatic presentations $[1,4]$.

However, cases of metastatic progression in the brain site are extremely rare and of clinical relevance, as they represent diseases that responded for a longer period to systemic treatment, an uncommon characteristic of this neoplasm, since in the vast majority of cases the associated early mortality does not allow such progression, about $0.1 \%$ to $0.3 \%$ of all cases [8].

The present study, in essence, aims to report a rare case of a patient diagnosed with pancreatic cancer, which had a median overall survival significantly higher than the median time related to this neoplasia, reaching more than 48 months of metastatic disease and being submitted to five lines of cancer treatment performed and consequently, presenting during the final course of the disease an unusual progression associated with symptomatic brain metastatic activity.

Thus, we correlated the relevant literary data with the events presented by the reported patient.

\section{Case report}

We reported a female patient, diagnosed with pancreatic adenocarcinoma stage IV, associated with metastatic to the pulmonary and hepatic sites. The patient presented primary nodular lesion in the pancreatic tail $(4.5 \times 3.9 \times 3.1 \mathrm{~cm})$ compromising splenic vascularization, presence of indeterminate pulmonary micronodules and the largest liver lesions in hepatic segment VIII $(4.3 \times 4.3 \mathrm{~cm})$, and CA marker initial 19-9: 19,670, which characterizes a high volume of disease initially.

The patient underwent chemotherapy based on the FOLFIRINOX regimen (Fluorouracil, Leucovorin, Irinotecan and Oxaliplatin), with maintenance management PANOPTIMOX Trial, performing 28 cycles, with a treatment pause due to a cholicystectomy procedure, later as FOLFOX (Fluorouracil, Leucovorin, Oxaliplatin) as second-line chemotherapy was instituted, being carried out 5 cycles.

After local disease progression, chemotherapy based on antimicrotubule / taxane agent: paclitaxel (the drug nabpaclitaxel was not used due to lack of access) and gemcitabine in six chemotherapy cycles were instituted, a maintenance period with Cabecitapine was instituted, after a new progression 
in the pancreatic and hepatic site when the FOLFIRI (Fluorouracil, Leucovorin, Irinotecan), carried out 16 chemotherapy cycles, thus instituting the fourth line of systemic treatment, based on the XELOX re-induction scheme (Oxaliplatin, Capecitabine) with new progression of associated disease, when defined as the best exclusive supportive care.

During the period of palliative treatment already under exclusive clinical support, the patient was brought to the hospital on an emergency basis due to severe headache associated with sudden paralysis in the right hemibody with a brachial predominance.

Complementary imaging exams showed progression of metastatic disease in the central nervous system with multiple lesions and major vasogenic edema both by computed tomography without contrast and by magnetic resonance imaging (RMI) of the skull presenting a solid, expansive centered lesion $(6.3 \times 2.9 \mathrm{~cm})$ in the left Parieto-occipital sulcus region, associated with perilesional vasogenic edema, with diffuse alteration of the grooves between the ipsilateral cerebral hemispheric cortical gyres, contralateral collapse related to 0.6 midline deviation $\mathrm{cm}$ (Figure 1).

Due to the RPA (Replication Protein A) classified as 3 unfavorable, it was decided not to intervene in the new disease progression, since the patient remained being cared for and died after the diagnosis of brain metastases, several years since the diagnosis and beginning treatment for advanced pancreatic cancer.

The patient reported in this case was followed up during the treatment, when she was diagnosed with stage IV pancreatic adenocarcinoma, including the period when she and her family opted for excluded palliative care when there was progression in the brain site, until the death of the patient. 

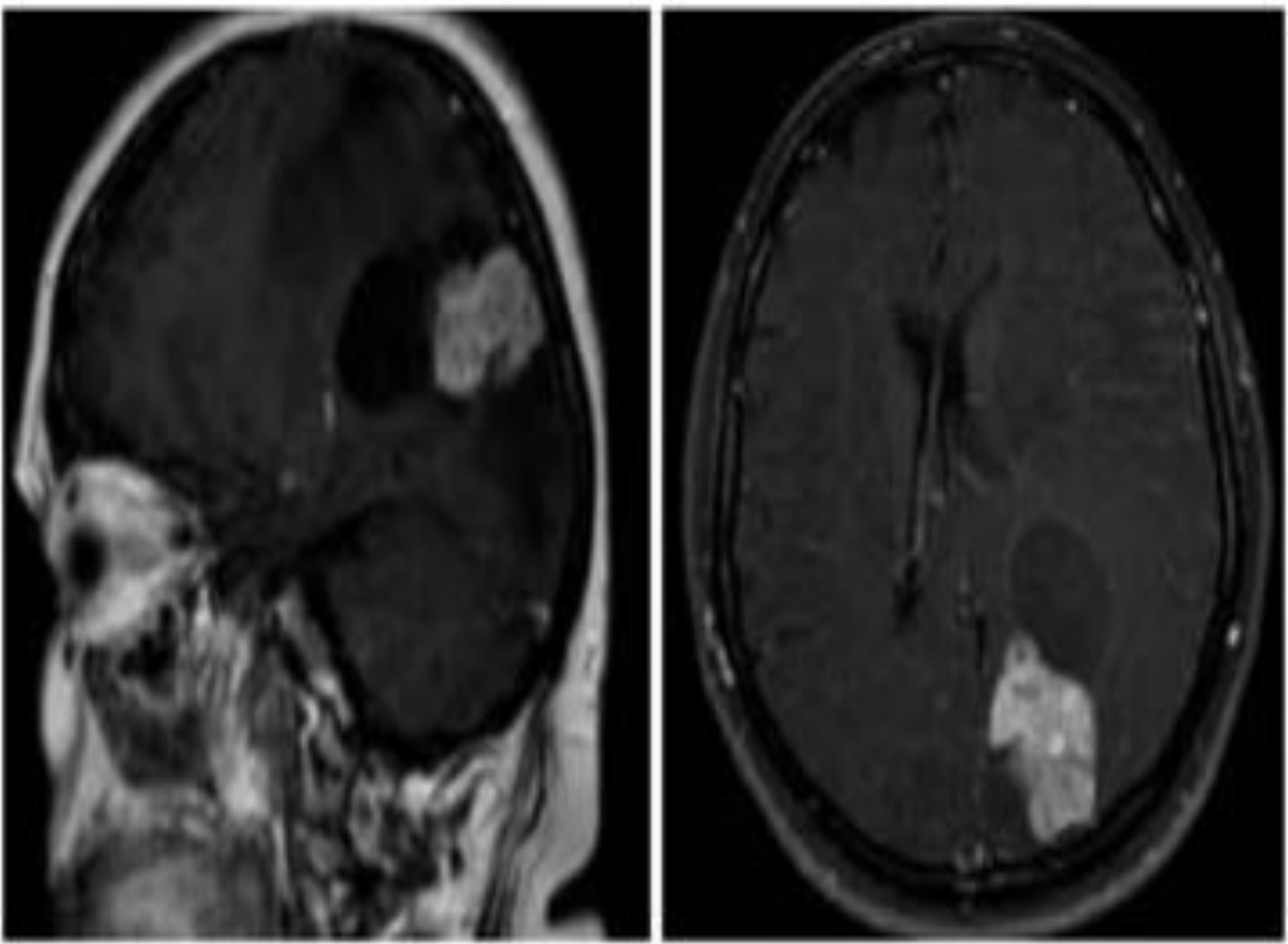

Figure 1. RMI: Solid, expansive lesion $(6.3 \times 2.9 \mathrm{~cm})$ in the left parieto-occipital sulcus region, associated with perilesional vasogenic edema and contralateral collapse related to 0.6 midline deviation $\mathrm{cm}$.

\section{Discussion and Conclusion}

Pancreatic cancer, a neoplastic entity represented by the subtype Adenocarcinoma acinar in $90 \%$ to $95 \%$ of cases, with a predilection for males, older than 60 years and for black ethnicity, tumor subtype intrinsically associated with somatic causes, especially smoking, obesity and alcoholism [1-3,5,7].

However, genetic causes such as Peutz-Jeghers syndrome and hereditary pancreatitis, as well as germline mutations represented by changes in
BRCA1， BRCA2, p16, ATM, STK11, PRSS1, PRSS 2, SPINK1 and PALB2 are associated with carcinogenic activity pancreatic disease in about $5 \%$ of cases [4-5].

According to the National Cancer Institute (NCI), in Brazil such a carcinogenic subtype is responsible for about $2 \%$ of all diagnoses among solid tumors and responsible for $4 \%$ of neoplastic mortality [2].

According to a European epidemiological study, pancreatic cancer represents fourth mortality among solid tumors, surpassed only by 
lung, colorectal and prostate cancer in males and breast, colorectal and lung cancer in females [5-6].

Solid tumor composed of similar incidence and mortality rates, with life expectancy being approximately $5 \%$ in 5 years, the diagnosis in most cases is late, in advanced stages strengthening the presentations of associated metastatic activities [1,4].

About $15 \%$ to $20 \%$ of all patients are eligible for the surgical procedure, of which the average survival is around 5 years for about $25 \%$ of the patients who do not have lymph node involvement and $10 \%$ when such involvement is evidenced [3].

Pancreatic neoplasia has a tendency to spread early to the liver and peritoneum, and can be found in pulmonary and bone metastasis $[1,3,5-$ 7]. However, the presence of brain metastasis is a rare event and never seen before in our service that treats a high volume of pancreatic neoplasms annually. Some cases of prolonged survival in advanced pancreatic adenocarcinoma have been reported, and it is possible to reach close to 60 months.

The prognosis associated with pancreatic cancer is still considered reserved, since it consists of an average survival rate between 8 to 12 months for locally advanced disease and up to 6 months when metastatic activity is evidenced [3].

According to the phase III Polo study, it was shown that about $4 \%$ to $5 \%$ of patients have germline mutations in $B R C A 1$ or $B R C A 2$, which undergo platinum-based chemotherapy in the first line, without associated disease progression, were randomized, and candidates for maintenance treatment with PARP inhibitors, and such patients were submitted to treatment with olaparib versus placebo, obtained a significant progressive survival gain, about 7.4 months compared to just 3.8 months respectively [9].

Despite the improvement in clinical research in recent years, the data previously reported confirm the characteristic associated with one of the most aggressive cancers in the history of clinical oncology under study. Emphasizing that our patient did not have access to the most recently incorporated interventions in the area, such as the chemotherapy drug nab paclitaxel or the medication olaparib, and despite these deficiencies she had a survival rate eight times higher than expected for her disease.

Cases of metastatic progression in the brain site due to pancreatic cancer being treated are extremely rare and of clinical relevance, since in the vast majority of cases the associated mortality does not allow such 
progression, occurring in about $0.1 \%$ to $0.3 \%$ of all cases [8].

The greatest relevance of these cases is not only in reporting the involvement of the central nervous system, but mainly in citing cases with much longer-than-usual survival and their treatment sequences. Only 6 studies were found in a scientific review of the topic, in which it was possible to show metastatic progression activity, which is similar to the data presented about the studied patient.

A Brazilian study showed only one case related to a male patient, aged over 50 years, who triggered acute dysgraphia and speech apraxia, came to the emergency room, with magnetic resonance imaging showed a lesion in the pancreatic body / tail compatible with Anatomopathological carcinoma, and lesion in the adrenal and cerebral regions of a metastatic order, in this case the opening of the picture with brain disease is probably due to the location of the primary lesion in the body / tail of the pancreas, which confers a later diagnosis and an even more dismal prognosis [10].

Asian case report, confirmed diagnosis of pancreatic cancer, T3N3M1, stage: IV, metastatic in brain site, in a male patient at 68 years of age, due to abrupt change in mental state and gait in a patient previously without a diagnosis of cancerous disease [11].
Studies on diagnostic evidence in an advanced stage of ongoing metastatic disease. The study with the largest retrospective production is a Germanic case series, which showed a total of 12 cases of patients with pancreatic cancer who developed metastasis in the brain region. Of these, only one female patient, among all 3 cases showed metastatic activity associated with the diagnosis. Since 7 patients had a single brain metastasis, others at the reported site, disease progression at the hepatic and pulmonary sites were frequently presented by the reported patients [12].

An American patient, male, 57 years old, with sports background, was admitted to an intensive care unit after loss of consciousness and sensory degradation. Since the same after initial exams, he was diagnosed with an expansive, infiltrating lesion, typically cancerous in the pancreatic region associated with multiple metastatic activities in the brain site associated with leptomeniginous, pulmonary and hepatic carcinomatosis, thus being instituted an exclusively palliative treatment [13].

A rare case report of an American patient, female, 69 years old, with a history of Lynch syndrome and colon cancer (2006), was diagnosed with pancreatic adenocarcinoma (2015), and developed a single metastatic brain lesion secondary to pancreatic cancer 
(2017) [8].

An Asian study reported 2 cases of pancreatic cancer associated with metastatic to the brain site at diagnosis, both in male patients older than 55 years and with lymph node and liver involvement [14].

It clearly calls attention to the rapid progression associated with the often-late diagnosis of pancreatic cancer. In addition, the data in which only 2 reports with female patients were found, which presented such associated metastatic involvement and most often a late involvement in the course of the central nervous system disease with a clear tendency to palliative treatment exclusive support in this context. Thus, agreeing with the aforementioned literary data about the rarity of the case being presented.

Thus, a clinical case becomes relevant due to the rare manifestation of metastasis in the brain site secondary to the pancreatic tumor, which is very little documented in the literature, about $0.1 \%$ per year. $0.3 \%$ of cases [8].

In addition, the survival shown by the patient to the metastatic diagnosis is remarkable, four lines of systemic treatment, for 4 years, an unusual period related to the subtype and tumor stage initially diagnosed, restricted in such a stage the average survival around 6 months, due to extremely

aggressive

disease progression $[3,6-7,9]$.

\section{References}

[1] Ducreux M, Cuhna AS, Caramella C, Hollebecque A, Burtin P, Goéré D, Seufferlein T, Haustermans K, Van Laethem JL, Conroy T, Arnold D; ESMO Guidelines Committee. Cancer of the pancreas: ESMO Clinical Practice Guidelines for diagnosis, treatment and follow-up. Ann of Oncol. 2015;26(5668). doi:10.1093/annonc/mdv295.

[2] National Cancer Institute. Ministry of Health. Health Care Secretariat. Estimates 2008: cancer incidence in Brazil. Rio de Janeiro: INCA; 2007.

[3] Silva CSHA da, Lucas SFLM, Nakatsu E, Moricz A, Silva RA, Pacheco Jr AM, Campo T. Pancreatic adenocarcinoma in a young patient: case report. Arch Med Hosp Fac Cienc Med Santa Casa São Paulo. 2011; 56(1):36-9.

[4[ Malvezzi M, Bertuccio P, Levi F, La Vecchia C, Negri E. European cancer mortality predictions for the year 2014. Ann Oncol. 2014; 25(8): 1650-6. https://doi.org/10.1093/annonc/mdu138.

[5] Yeo TP. Demographics, epidemiology, and inheritance of pancreatic ductal adenocarcinoma. Semin Oncol. 2015; 42(1):8-18. https://doi.org/10.1053/j.seminoncol.201 4.12.002.

[6] Siegel RL, Miller KD, Jemal A. Cancer statistics, 2019. CA Cancer J Clin. 2019; 69(1):7-34. doi: 10.3322/caac. 21551 . 
[7] Maisonneuve P, Lowenfels AB. Risk factors for pancreatic cancer: a summary review of meta-analytical studies. Int $\mathrm{J}$ Epidemiol. $\quad 2015 ; 44(1): \quad$ 186-98. https://doi.org/10.1093/ije/dyu240.

[8] Dalal K, Khrizman P, Chaaya, AA. Isolated Brain Metastasis From Pancreatic Cancer in Lynch Syndrome. Am J Gastroenterol. 2019;114(1):S697. doi: 10.14309/01.ajg.0000594536.59856.1f

[9] Golan T, Hammel P, Reni M, Van Cutsem E, Macarulla T, Hall MJ, Park JO, Hochhauser D, Arnold D, Oh DY, Reinacher-Schick A, Tortora G, Algül H, O'Reilly EM, McGuinness D, Cui KY, Schlienger K, Locker GY, Kindler HL. Maintenance Olaparib for Germline BRCA-Mutated Metastatic Pancreatic Cancer. N Engl J Med. 2019; 381(4):31727. doi:10.1056/nejmoa1903387

[10] Orsini M, Sarmet A, Consídera D, Barbosa MD, Nascimento LM, Leite MAA, Souza JA. Brain metastases in pancreatic carcinoma: a rare situation. Rev Bras Neurol. 2014;50(4):88.

[11] Matsumoto H, Yoshida Y. Brain metastasis from pancreatic cancer: a case report and literature review. Asian J Neurosurg. $\quad 2015 ; 10(1): 35-9 . \quad$ doi: 10.4103/1793-5482.151507

[12] Lemke J, Scheele J, Kapapa T, Wirtz CR, Henne-Bruns D, Kornmann M. Brain metastasis in pancreatic cancer. Int J Mol Sci. 2013 Feb 19;14(2):4163-73. doi: 10.3390/ijms14024163.

[13] Rao R, Sadashiv SK, Goday S, Monga D. An extremely rare case of pancreatic cancer presenting with leptomeningeal carcinomatosis and synchronous intraparenchymal brain metastasis. Gastrointest Cancer Res. 2013 May;6(3):90-2.

[14] Kuratsu J, Murakami M, Uemura S, Ushio Y. Brain and skull metastases of hepatic or pancreatic cancer--report of six cases. Neurol Med Chir (Tokyo). 1990 Jul;30(7):476-82. doi: 10.2176/nmc.30.476.

Conflict of interest: The author declares no conflicts of interest.

Acknowledgements: We thank very much the whole team for the excellent multidisciplinary management of the case and the patient and or the responsible person for having trusted in our work and accepting the publication of this case report.

Funding: The author declares no funding to develop this article.

How to cite this article: Pastorello J, Lando E, Bueno MR, Amaral CS. Metastatic brain tumor secondary to pancreatic adenocarcinoma. Brazilian Journal of Case Reports. 2022 JanMar;02(1):32-39. 